\title{
The ethics of anonymised HIV testing of pregnant women: a reappraisal
}

Anthony J Pinching St Bartholomew's and The Royal London School of Medicine and Dentistry, London

Dr de Zulueta articulates some important and commonly held concerns about the anonymised screening programme for HIV in pregnant women, which is one of a number of such programmes that are current. However, in my view, many of these concerns reflect a failure to understand two key distinctions.

In both these regards, there is a danger of putting up a "straw man" for challenge. In this commentary, I wish to pick up some of these issues to help to resolve the apparent ethical tensions that Dr de Zulueta has set out.

Firstly, the unlinked (anonymised) seroprevalence surveillance (screening) programmes, including that on pregnant women, comprise research studies that are designed to inform policy and practice, as well as individual decision making. They are not screening for the purpose of clinical care. The anonymisation is a procedural research tool, which enables the studies to be done in a way that does not undermine consent or individual autonomy. The ethical standards and duties expected of professionals are thus, in this specific regard, those that relate to the conduct of clinical research. That research is evidently designed to improve the quality of clinical care as a result of the information and insight gained. The duty of care is to apply the results of properly conducted and valid research in order to improve clinical practice on behalf of the individuals receiving care, in this case the mother and unborn child. The research conducted, in this case by a particular form of screening procedure, is not the same as a screening procedure done for clinical purposes to identify individuals who could benefit from an intervention. The ethical debate must therefore focus on the purpose in applying the procedure, not on the procedure itself, regardless of intent.

Secondly, it is vital to distinguish, in debating such issues of principle, between a fundamentally unsound policy or practice on the one hand, and poor standards of implementation of that policy or practice on the other. Some of the aspects of the case studies blur these boundaries and are in danger of skewing our understanding of the underlying issues.

It is sobering to recall that the implementation of the unlinked anonymised surveillance programmes in the UK were delayed for some time because of the cogently argued view of an influential ethicist, Professor Ian Kennedy. He convinced most of the UK Social Services Select Committee in 1987 that there are some studies that, however important, cannot be done if they infringe basic human rights. Fortunately, it was possible to satisfy most commentators and politicians that the way in which the studies were done could avoid the fundamental concern which he was expressing.

A key part of this was to ensure that procedures were explicitly in place to enable women for whom HIV testing was relevant to their personal decision making in the antenatal context, to access such testing on a voluntary named basis. For this reason, posters and supporting literature, together with staff training in relation to the surveillance study, specifically set out the availability of such tests and the need to discuss the issues with midwives, doctors or others involved in antenatal care. The second component to deal with the expressed concerns was to make it very clear that, even though the results could not be linked back to the individual, individuals could elect not to participate in the programme. Despite these safeguards, it is evident that much misunderstanding about the nature and process of the unlinked seroprevalence programmes exists, as described and indeed exemplified by Dr de Zulueta.

As a result of the implementation of these and other surveillance studies, it was possible to identify the extent of HIV infection in a variety of high and low prevalence settings. Above all, it was possible to obtain an estimate of the discrepancy between the prevalence in the population being studied and the known rates in the same settings 
from voluntary named testing. I would argue that it is exactly because we have this information that we have been able to shape and refine clinical practice to the benefit of individuals. Above all, it can help ensure that those who are at risk have the opportunity to obtain testing, where it is of value in informing their care and that of the unborn child.

It has also been possible to focus resources where the discrepancy is greatest, for example in areas of East London, where I practise. The studies have also demonstrated that the proportional discrepancy between named testing and the surveillance data is indeed sometimes even greater in areas that are perceived to be lower prevalence, even though the absolute numbers are relatively smaller. Again, this has provided exactly the sort of information that Dr de Zulueta seems to want all women and clinicians to have as part of their individual decision making.

Thus at no time have clinicians been required or encouraged to "refrain from informing pregnant women of the benefits of voluntary named testing" in relation to the unlinked seroprevalence studies. Indeed the contrary is the case, since seroprevalence studies have drawn attention to the issue in general, both for patients and clinicians, as well as providing data to inform decision making in specific settings. The HIV seroprevalence data obtained using Guthrie test cards also ensures that coverage is at all centres, not just those participating in the antenatal studies.

\section{Very real problems}

The benefits of knowing HIV status in this setting have undoubtedly increased. Yet they may not, for all individuals, outweigh the hazards. The risks cannot be lightly swept aside because of the apparent generality of benefit. Sadly, in many disadvantaged communities, especially some ethnic minority cultures, very real problems continue, though they are not the same as those that were more evident earlier in the epidemic to which Dr de Zulueta refers. For example, the safety and security of the mother is of vital interest to the unborn child. Ultimately the choice to make informed decisions about medical interventions remains the proper right and prerogative of the pregnant woman. To acknowledge and enable this is not to paternalise, but to provide opportunity for individuals to exert their autonomy. To deny choice because of a seemingly obvious benefit to the unborn child is surely paternalistic. These considerations are as true for screening tests for Down's syndrome or other congenital infections as they are for HIV. This is "normal" and good practice, even though it does allow people to make decisions that might not receive universal agreement and that might even by judged not to be in the best interests of the unborn child.

\section{Poor clinical practice}

In the clinical scenarios, there are several examples of poor clinical practice. The midwife's reported response to Frances was evidently inappropriate. It would be unprofessional to be visibly "shaken" or indeed surprised by Frances's concerns; her response suggests poor training. A person who wished to have a voluntary named test should be offered this, without constraint and in as enabling a way as possible. This would include ensuring immediate counselling and advice by the midwife to the extent of her training and ability, and/or onward referral, sensitively explained, to one of a number of individuals or settings where further skills and expertise are available. Sexually transmitted Disease (STD) clinics are only one of a number of such settings. Clinics doing unlinked surveillance schemes would have had clear guidance on the options. The reported response to Frances's wish to have voluntary named testing and to know the result is a non sequitur, in that it asks for her altruistic participation in the surveillance study, as if this was an alternative, as opposed to a both/and option. Her right to refuse participation in the research study was evidently a quite separate issue from whether or not she had her own test. It would be interesting to know what the "principle" was that made Frances refuse. If it was unwillingness to participate because of the approach taken by the midwife, it is understandable, though scarcely an issue of principle!

In the second scenario, it would seem that Emma has limited awareness about HIV risks (which is sadly still widespread). It is the role of the information leaflets and the professionals involved in her care to do their best to ensure she is aware so that she can make an informed choice. The scenario seems to suggest that she had no professional guidance, which again represents a failure of normal professional duties of care. It has nothing intrinsically to do with the fact that the clinic was (apparently) also doing the unlinked seroprevalence study. If it were, at least it would have salvaged something from the errors or misjudgments, by providing the information about the discrepancy, which would, it is to be hoped, have led to better staff awareness and training. The case illustrates the need for continuing public education about HIV and emphasises the role of staff in individualising general health education messages. I would also argue that the mother does have some responsibility for equipping herself with relevant information and 
guidance to maximise the health opportunities for her unborn child.

We need also to be aware of how HIV is only one of a vast range of issues that women need to consider antenatally. The way in which this issue is addressed and "normalised" needs to ensure that the attention given to it is appropriate and proportionate, and does not, as it has in some instances, distract from other major issues.

This article, as with many others in this area, seems to assume that doing things for the public good is somehow opposed to individual benefit, rather than as well as such benefit. It also takes a rather static view of the process and seems not to acknowledge that the surveillance system has enabled us to gain the information to shape and evolve policies. Recent guidance to enhance antenatal testing for clinical benefit derives strongly from the information thereby obtained. New leaflets and guidance are now available that reflect the substantial shift in practice, perceptions and in opportunities to prevent vertical transmission.

The effectiveness of these changes in practice can, and indeed must, continue to be monitored. Unlinked surveillance is arguably the only means whereby we can demonstrate whether the move to increase the discussion of this issue, which Dr de Zulueta wishes to see, as I do, actually achieves the desired results. If so, it can be further refined in the light of the results. If not, the data would enable a critical evaluation of why or how the rhetoric of voluntary testing fails to achieve the desired outcomes. Thus we will all be wiser. To disallow it on what seem to be spurious ethical grounds seems unconscionable, and to fail to distinguish between research or evaluation and clinical practice.

Dr de Zulueta may feel that the data we now have on the epidemiology and the improved prospects for prevention, justify the introduction of screening for clinical benefit. Such a clinical screening programme could be considered justified for areas of high prevalence or of substantial discrepancy between open and anonymised positive tests, as determined by the surveillance data. Alternatively it could even be argued that it is justifiable to introduce it universally. Again, the arguments for or against would be informed by the surveillance data. The details of any such policy and its implementation (including the question of opting in or out), would merit careful professional and ethical scrutiny, as in the case of the unlinked surveillance, to maximise benefit and minimise harm. This is, however, a separate debate from whether the anonymised (unlinked) seroprevalence research studies are ethically acceptable, and whether they should continue.

Anthony 7 Pinching is Louis Freedman Professor of Immunology and Fellow, Department of Human Science and Medical Ethics, St Bartholomew's and The Royal London School of Medicine and Dentistry, Queen Mary \& Westfield College, West Smithfield, London EC1A $7 B E$ 